\title{
MATRIX TRANSFORMATIONS INVOLVING CERTAIN BANACH SPACE VALUED SEQUENCE SPACES
}

\author{
J. K. SRIVASTAVA AND B. K. SRIVASTAVA
}

\begin{abstract}
In this paper for Banach spaces $X$ and $Y$ we characterize matrix classes $(\Gamma(X, \lambda)$, $\left.l_{\infty}(Y, \mu)\right),(\Gamma(X, \lambda), C(Y, \mu)),\left(\Gamma(X, \lambda), c_{0}(Y, \mu)\right),\left(\Gamma(X, \lambda), \Gamma^{*}(Y, \mu)\right),\left(l_{1}(X, \lambda), \Gamma(Y, \mu)\right)$ and $\left(c_{0}(X, \lambda), c_{0}(Y, \mu)\right)$ of bounded linear operators involving $X$ - and $Y$-valued sequence spaces. Further as an application of the matrix class $\left(c_{0}(X, \lambda), c_{0}(Y, \mu)\right)$ we investigate the Banach space $B\left(c_{0}(X, \lambda), c_{0}(Y, \mu)\right)$ of all bounded linear mappings of $c_{0}(x, \lambda)$ to $c_{0}(Y, \mu)$.
\end{abstract}

\section{Introduction}

Let $\lambda=\left(\lambda_{k}\right)$ and $\mu=\left(\mu_{k}\right)$ be any sequences of non-zero complex numbers, $X$ and $Y$ be Banach spaces over the field $C$ of complex numbers and $B(X, Y)$ denote the Banach space of all bounded linear operators from $X$ into $Y$ with the usual operator norm.

Let $A=\left(A_{n k}\right),(n, k=1,2,3 \ldots)$ be an infinite matrix of bounded linear operators $A_{n k}$ on the Banach space $X$ into the Banach space $Y$. Then for the classes of $X$-valued sequences $E(X)$ and $Y$-valued sequences $F(Y)$, we define the matrix class $(E(X), F(Y))$ by saying that

$$
A=\left(A_{n k}\right) \in((E(X), F(Y))
$$

if

$$
\text { for every } \quad \begin{aligned}
\bar{x} & =\left(x_{k}\right) \in E(X), \\
y_{n} & =A_{n}(\bar{x})=\sum_{k=1}^{\infty} A_{n k} x_{k}
\end{aligned}
$$

converges in the norm of $Y$, for each $n$, and the sequence $\bar{y}=\left(y_{n}\right)=\left(A_{n}(\bar{x})\right)$ belongs to $F(Y)$. In such a case $\bar{y}=A \bar{x}$ is called the $A$ transform of $\bar{x}$.

We define

$$
\begin{aligned}
\Gamma(X, \lambda) & =\left\{\bar{x}=\left(x_{k}\right): x_{k} \in X,\left\|\lambda_{k} x_{k}\right\|^{1 / k} \rightarrow 0 \text { as } k \rightarrow \infty\right\}, \text { and } \\
\Gamma^{*}(X, \lambda) & =\left\{\bar{x}=\left(x_{k}\right): x_{k} \in X, \sup _{k}\left\|\lambda_{k} x_{k}\right\|^{1 / k}<\infty\right\} .
\end{aligned}
$$

Received December 15, 1997; revised September 8, 1999.

2000 Mathematics Subject Classification. 46A45, 40H05.

Key words and phrases. Vector sequence spaces, bounded linear transformations, matrix transformations. 
Similarly $\Gamma(Y, \mu)$ and $\Gamma^{*}(Y, \mu)$ are defined.

The above defined spaces are the generalizations of $\Gamma$ and $\Gamma^{*}$ respectively, introduced by Iyer $[2,3]$. Moreover $\Gamma(X, \lambda)$ and $\Gamma^{*}(X, \lambda)$ can be viewed as special cases of $c_{0}(X, \lambda, p)$ and $l_{\infty}(X, \lambda, p)$ respectively introduced and studied in $[8,9]$. In fact $c_{0}(X, \lambda,(1 / k))=$ $\Gamma(X, \lambda)$ and $l_{\infty}(X, \lambda,(1 / k))=\Gamma^{*}(X, \lambda)$. However we prefer the notations $\Gamma(X, \lambda)$ and $\Gamma^{*}(X, \lambda)$ in place of $c_{0}(X, \lambda,(1 / k))$ and $l_{\infty}(X, \lambda,(1 / k))$ for obvious reasons as we observe that they are more close to $\Gamma$ and $\Gamma^{*}$. Analogous to $P_{\lambda, p}$ for $c_{0}(X, \lambda, p)$ of $[8]$ we define

$$
P_{\lambda}(\bar{x})=\sup _{k}\left\|\lambda_{k} x_{k}\right\|^{1 / k}, \quad \bar{x}=\left(x_{k}\right) \in \Gamma(X, \lambda) \text { or } \Gamma^{*}(X, \lambda),
$$

and we see that $\left(\Gamma(X, \lambda), P_{\lambda}\right)$ is a complete total paranomed space [cf. Theorem 2.22 of [9]] and hence $\Gamma(X, \lambda)$ is a complete linear metric space with respect to the metric induced by the paranorm. $\bar{\theta}=(\theta, \theta, \theta, \ldots)$ will denote the zero of $\Gamma(X, \lambda)$. Some other properties such as generalized Köthe-Toeplitz duals, continuous duals etc. for the space $\left(\Gamma(X, \lambda), P_{\lambda}\right)$ can easily be derived from the concerning results already investigated in [8] about $c_{0}(X, \lambda, p)$. Since $\inf _{k} 1 / k=0$, therefore $\Gamma^{*}(X, \lambda)$ is simply a complete metric space with respect to the metric induced by $P_{\lambda}$. This assertion follows from Theorem 2.18 of [9]. We further define

$$
\begin{aligned}
& c_{0}(X, \lambda)=\left\{\bar{x}=\left(x_{k}\right): x_{k} \in X,\left\|\lambda_{k} x_{k}\right\| \rightarrow 0 \text { as } k \rightarrow \infty\right\}, \\
& c(X, \lambda)=\left\{\bar{x}=\left(x_{k}\right): x_{k} \in X, \text { there exists } l \in X \text { such that }\left\|\lambda_{k} x_{k}-l\right\| \rightarrow 0 \text { as } k \rightarrow \infty\right\}, \\
& l_{\infty}(X, \lambda)=\left\{\bar{x}=\left(x_{k}\right): x_{k} \in X, \sup _{k}\left\|\lambda_{k} x_{k}\right\|<\infty\right\}
\end{aligned}
$$

and $l_{1}(X, \lambda)=\left\{\bar{x}=\left(x_{k}\right): x_{k} \in X, \sum_{k=1}^{\infty}\left\|\lambda_{k} x_{k}\right\|<\infty\right\}$.

As mentioned in [8] and [9] we note these spaces are particular cases of $c_{0}(X, \lambda, p)$, $c(X, \lambda, p), l_{\infty}(X, \lambda, p)$ and $l(X, \lambda, p)$, when $p_{k}=1$ for all $k \geq 1$. Of course $c_{0}(X, \lambda)$, $c(X, \lambda), l_{\infty}(X, \lambda)$ are Banach spaces with the norm

$$
\|\bar{x}\|_{\lambda}=\sup _{k}\left\|\lambda_{k} x_{k}\right\|
$$

and $l_{1}(X, \lambda)$ is a Banach space with the norm

$$
\|\bar{x}\|_{\lambda}=\sum_{k=1}^{\infty}\left\|\lambda_{k} x_{k}\right\|
$$

We recall the definition of generalized $\beta$-dual for the class $E(X)$ of $X$-valued sequences.

Definition 1.1. [7, p.8] Let $X$ and $Y$ be Banach spaces and $\bar{A}=\left(A_{k}\right)$ a sequence of linear, but not necessarily bounded, operators $A_{k}$ on $X$ into $Y$. Suppose $E(X)$ is a 
non-empty set of $X$-valued sequences. Then the $\beta$-dual of $E(X)$ is defined by $E^{\beta}(X)=$ $\left\{\bar{A}=\left(A_{k}\right): \sum_{k=1}^{\infty} A_{k} x_{k}\right.$ converges in $Y$ for all $\left.\bar{x} \in E(X)\right\}$.

Definition 1.2. [7, p.5] Let $\bar{A}=\left(A_{k}\right)$ be a sequence in $B(X, Y)$. Then the group norm of $\left(A_{k}\right)$ is defined by $\left\|\left(A_{k}\right)\right\|=\sup \left\|\sum_{k=1}^{n} A_{k} x_{k}\right\|$, where supremum is taken over all $n \geq 1$ and $x_{k} \in S$, $(S$ is the closed unit sphere in $X)$.

We refer [7] for some of the properties of group norm and while dealing with group norm in section 4 , we shall use notation $R_{k}(\bar{A})=\left(A_{k}, A_{k+1}, \ldots\right)$.

A number of results regarding the characterization of matrix classes of linear operators between some of the Banach space valued sequence spaces corresponding to scalar sequence spaces $c_{0}, c, l_{\infty}, l_{p}$ etc. have been obtained, for instance see [7]. Our aim in this note is to investigate some of the matrix classes of linear operators involving certain Banach space valued sequence spaces which will lead to generalizations of some of the results determined by Rao $[5,6]$ for scalar sequence spaces $c_{0}, c, l_{1}, \Gamma, \Gamma^{*}$ etc.

\section{Matrix Transformations between Some Banach Space Valued Sequence Spaces}

In this section we characterize matrix transformations (of bounded linear operators) between certain Banach space valued sequence spaces, viz. $\left(\Gamma(X, \lambda), l_{\infty}(Y, \mu)\right),(\Gamma(X, \lambda)$, $c(Y, \mu)),\left(\Gamma(X, \lambda), c_{0}(Y, \mu)\right)$ and $\left(\Gamma(X, \lambda), \Gamma^{*}(Y, \mu)\right)$.

In order to establish conditions characterizing $\left(\Gamma(X, \lambda), c_{0}(Y, \mu)\right)$ we first prove:

Lemma 2.1. $\bar{A}=\left(A_{k}\right) \in \Gamma^{\beta}(X, \lambda)$, the generalized $\beta$-dual of $\Gamma(X, \lambda)$, if and only if

$$
\sup _{k}\left\|\lambda_{k}^{-1} A_{k}\right\|^{1 / k}<\infty .
$$

Proof. The sufficiency of the condition is straight forward. For the necessity, we suppose $\bar{A}=\left(A_{k}\right) \in \Gamma^{\beta}(X, \lambda)$ but $\sup _{k}\left\|\lambda_{k}^{-1} A_{k}\right\|^{1 / k}=\infty$. Then there exists a subsequence $(k(n))$ of $(k)$ such that $\left\|\lambda_{k(n)}^{-1} A_{k(n)}\right\|>n^{k(n)} ; n \geq 1$ and for each $n \geq 1$ there exists $z_{n} \in S$ (the closed unit sphere in $X$ ) such that $2\left\|\lambda_{k(n)}^{-1} A_{k(n)} z_{n}\right\|>n^{k(n)}$. Now we define

$$
\begin{aligned}
x_{k} & =n^{-k(n)} \lambda_{k(n)}^{-1} z_{n} ; \quad \text { if } k=k(n), \quad n \geq 1 \quad \text { and } \\
& =\theta, \quad \text { otherwise. }
\end{aligned}
$$

Then we see that $\bar{x}=\left(x_{k}\right) \in \Gamma(X, \lambda)$ but $\bar{A}=\left(A_{k}\right) \notin \Gamma^{\beta}(X, \lambda)$, which leads to a contradiction. This completes the proof.

Theorem 2.2. $A=\left(A_{n k}\right) \in\left(\Gamma(X, \lambda), l_{\infty}(Y, \mu)\right)$ if and only if there exists $M>0$ such that

$$
\left\|\mu_{n} \lambda_{k}^{-1} A_{n k}\right\|^{1 / k} \leq M
$$


independently of $n \geq 1$ and $k \geq 1$.

Proof. For the sufficiency, we consider $\bar{x}=\left(x_{k}\right) \in \Gamma(X, \lambda)$ and for $\varepsilon>0$ we choose $0<\eta<1$ so that $\eta M<\frac{\varepsilon}{3+\varepsilon}$. Then there exists a $K$ such that $\left\|\lambda_{k} x_{k}\right\|^{1 / k}<\eta$ for all $k \geq K$.

Now for $y_{n}=\sum_{k=1}^{\infty} A_{n k} x_{k}$,

$$
\begin{aligned}
\left\|\mu_{n} y_{n}\right\| & =\left\|\sum_{k=1}^{\infty} \mu_{n} A_{n k} x_{k}\right\| \\
& \leq \sum_{k=1}^{K-1} M^{k}\left\|\lambda_{k} x_{k}\right\|+\frac{M \eta}{1-M \eta} \\
& <\sum_{k=1}^{K-1} M^{k}\left\|\lambda_{k} x_{k}\right\|+\varepsilon,
\end{aligned}
$$

which implies that $y_{n}$ exists for each $n \geq 1$. Moreover

$$
\sup _{n}\left\|\mu_{n} y_{n}\right\|<\infty
$$

and so $\bar{y}=\left(y_{n}\right) \in l_{\infty}(Y, \mu)$.

For the necessity, suppose $A=\left(A_{n k}\right) \in\left(\Gamma(X, \lambda), l_{\infty}(X, \lambda)\right)$. Then for every $\bar{x}=$ $\left(x_{k}\right) \in \Gamma(X, \lambda), y_{n}=\sum_{k=1}^{\infty} A_{n k} x_{k}$ exists for each $n \geq 1$, and $\sup _{n}\left\|\mu_{n} y_{n}\right\|<\infty$. Thus by Lemma 2.1 we have that

$$
\sup _{k}\left\|\mu_{n} \lambda_{k}^{-1} A_{n k}\right\|^{1 / k}<\infty, \quad \text { for each } n \geq 1 .
$$

Now we denote

$$
f_{n}(\bar{x})=\left\|\sum_{k=1}^{\infty} \mu_{n} A_{n k} x_{k}\right\|
$$

and

$$
\sup _{k}\left\|\mu_{n} \lambda_{k}^{-1} A_{n k}\right\|^{1 / k}=L(n), \quad n \geq 1 .
$$

Then we see that $f_{n}(\bar{x})$ is pointwise bounded functional as $\bar{y}=\left(y_{n}\right) \in l_{\infty}(Y, \mu)$. Further for sufficiently small $\eta>0$ if $P_{\lambda}(\bar{x})=\sup _{k}\left\|\lambda_{k} x_{k}\right\|^{1 / k}<\eta$, for $\bar{x}=\left(x_{k}\right) \in \Gamma(X, \lambda)$ then we have

$$
\begin{aligned}
f_{n}(\bar{x}) & =\left\|\sum_{k=1}^{\infty} \mu_{n} A_{n k} x_{k}\right\| \\
& \leq \sum_{k=1}^{\infty}\left\|\mu_{n} \lambda_{k}^{-1} A_{n k}\right\|\left\|\lambda_{k} x_{k}\right\| \\
& <\frac{L(n) \eta}{1-L(n) \eta}
\end{aligned}
$$


which implies that for each $n \geq 1 f_{n}$ is continuous on $\left(\Gamma(X, \lambda), P_{\lambda}\right)$. Since $\left(\Gamma(X, \lambda), P_{\lambda}\right)$ is a complete linear metric space therefore by Osgood theorem (a verson of uniform boundedness principle) there exists $L>0$ and a closed sphere which can without loss of generality be taken as $S[\bar{\theta}, \delta]$ in $\Gamma(X, \lambda)$ with centre $\bar{\theta}$ and radius $\delta$ such that

$$
\left|f_{n}(\bar{x})\right| \leq L
$$

for all $\bar{x}=\left(x_{k}\right) \in S[\bar{\theta}, \delta]$ and for all $n \geq 1$. Now for arbitrary $z \in S$ and $k \geq 1$ we take $\bar{x}=\left(\theta, \theta, \ldots \theta, \delta^{k} \lambda_{k}^{-1} z, \theta, \theta \ldots\right) \in S[\bar{\theta}, \delta]$ where $\delta^{k} \lambda_{k}^{-1} z$ is at the $k^{t h}$ place. Thus by (2.1) we have

$$
\left\|\mu_{n} \lambda_{k}^{-1} \delta^{k} A_{n k} z\right\| \leq L
$$

for all $z \in S$ and for all $n \geq 1$ and $k \geq 1$, and hence

$$
\left\|\mu_{n} \lambda_{k}^{-1} A_{n k}\right\|^{1 / k} \leq \frac{L^{1 / k}}{\delta} \leq M,
$$

for all $n \geq 1$ and $k \geq 1$, where $M=\delta^{-1} \max (1, L)$. This completes the proof.

Theorem 2.3. $A=\left(A_{n k}\right) \in(\Gamma(X, \lambda), c(Y, \mu))$ if and only if

(i) there exists $M>0$ such that

$$
\left\|\mu_{n} \lambda_{k}^{-1} A_{n k}\right\|^{1 / k} \leq M
$$

independently of $n \geq 1$ and $k \geq 1$; and

(ii) $\lim _{n} \mu_{n} A_{n k}$ exists for each $k \geq 1$.

Proof. Let (i) and (ii) hold and $\bar{x}=\left(x_{k}\right) \in \Gamma(X, \lambda)$. Now for $0<\epsilon<1$ choose $\eta$ so that $0<\eta M<\frac{\varepsilon}{3+\varepsilon}$. Then there exists $K$ such that

$$
\left\|\lambda_{k} x_{k}\right\|^{1 / k}<\eta, \quad \text { for all } k \geq K .
$$

Now

$$
\left\|\mu_{n} y_{n}\right\| \leq \sum_{k=1}^{K-1} M^{k}\left\|\lambda_{k} x_{k}\right\|+\frac{M \eta}{1-M \eta}<\infty .
$$

Thus $y_{n}$ exists for each $n \geq 1$. Further $\lim _{n} \mu_{n} A_{n k} x$ exists for each $k$ and each $x \in X$, so we take

$$
\lim _{n} \mu_{n} A_{n k} x=A_{k} x
$$

for each $k \geq 1$ and $x \in X$. By Banach-Steinhaus theorem, $A_{k} \in B(X, Y)$ for each $k \geq 1$. Thus form (i) and (ii) we easily get that

$$
\left\|\lambda_{k}^{-1} A_{k}\right\|^{1 / k} \leq M, \quad \text { for all } k \geq 1 .
$$


Now using (2.2) we easily see that $l=\sum_{k=1}^{\infty} A_{k} x_{k}$ exists in $Y$. Thus

$$
\begin{aligned}
\left\|\mu_{n} y_{n}-l\right\| & \leq \sum_{k=1}^{K-1}\left\|\left(\mu_{n} A_{n k}-A_{k}\right) x_{k}\right\|+\sum_{k=K}^{\infty}\left\|\mu_{n} \lambda_{k}^{-1} A_{n k}\right\|\left\|\lambda_{k} x_{k}\right\|+\sum_{k=K}^{\infty}\left\|\lambda_{k}^{-1} A_{k}\right\|\left\|\lambda_{k} x_{k}\right\| \\
& \leq \sum_{k=1}^{K-1}\left\|\left(\mu_{n} A_{n k}-A_{k}\right) x_{k}\right\|+2 \frac{M \eta}{1-M \eta} \\
& <\frac{\varepsilon}{3}+\frac{2 \varepsilon}{3}=\varepsilon
\end{aligned}
$$

for all sufficiently large values of $n$ and so $y=\left(y_{n}\right) \in c(Y, \mu)$.

Necessity of (ii) can easily be proved by considering the sequence $\bar{x}=(\theta, \theta, \theta, \ldots, \theta, x$, $\theta, \theta)$ taking $x \in X$ at $k^{t h}$ place, $k \geq 1$. Moreover since $(\Gamma(X, \lambda), c(Y, \mu)) \subset(\Gamma(X, \lambda)$, $\left.l_{\infty}(Y, \mu)\right)$ therefore the necessity of (i) follows from Theorem 2.2, this completes the proof.

Theorem 2.4. $A=\left(A_{n k}\right) \in\left(\Gamma(X, \lambda), c_{0}(Y, \mu)\right)$ if and only if

(i) there exists $M>0$ such that

$$
\left\|\mu_{n} \lambda_{k}^{-1} A_{n k}\right\|^{1 / k} \leq M
$$

independently of $n \geq 1$ and $k \geq 1$; and

(ii) $\lim _{n} \mu_{n} A_{n k}=\theta$, for each $k$

Proof. It can easily be proved along the lines of Theorem 2.3.

Theorem 2.5. $A=\left(A_{n k}\right) \in\left(\Gamma(X, \lambda), \Gamma^{*}(Y, \mu)\right)$ if and only if there exists $M>0$ such that

$$
\left\|\mu_{n} \lambda_{k}^{-1} A_{n k}\right\|^{1 /(n+k)} \leq M
$$

independently of $n \geq 1$ and $k \geq 1$.

Proof. For the sufficiency of the condition, consider $\bar{x}=\left(x_{k}\right) \in \Gamma(X, \lambda)$ and $\varepsilon>0$ and choose $\eta$ so that $0<\eta M<\frac{\varepsilon}{1+\varepsilon}$. Then there exists $K$ such that $\left\|\lambda_{k} x_{k}\right\|^{1 / k}<\eta$, for all $k \geq K$. Now

$$
\begin{aligned}
\left\|\mu_{n} y_{n}\right\| & \leq \sum_{k=1}^{K-1} M^{n+k}\left\|\lambda_{k} x_{k}\right\|+\sum_{k=K}^{\infty} M^{n+k} \eta^{k} \\
& <M^{n}\left\{\sum_{k=1}^{K-1} M^{k}\left\|\lambda_{k} x_{k}\right\|+\varepsilon\right\}<\infty .
\end{aligned}
$$

Thus $y_{n}$ exists for each $n \geq 1$. Further

$$
\begin{aligned}
\left\|\mu_{n} y_{n}\right\|^{1 / n} & \leq M\left\{\sum_{k=1}^{K-1} M^{k}\left\|\lambda_{k} x_{k}\right\|+\varepsilon\right\}^{1 / n} \\
& \leq M \max \left\{1, \sum_{k=1}^{K-1} M^{k}\left\|\lambda_{k} x_{k}\right\|+\varepsilon\right\}
\end{aligned}
$$


and hence $\sup _{n}\left\|\mu_{n} y_{n}\right\|^{1 / n}<\infty$. Thus $y=\left(y_{n}\right) \in \Gamma^{*}(Y, \mu)$.

Conversely let $A=\left(A_{n k}\right) \in\left(\Gamma(X, \lambda), \Gamma^{*}(Y, \mu)\right)$. Then $y_{n}=\sum_{k=1}^{\infty} A_{n k} x_{k}$ exists for each $n \geq 1$ and for each $\bar{x}=\left(x_{k}\right) \in \Gamma(X, \lambda)$, and $\sup _{n}\left\|\mu_{n} y_{n}\right\|^{1 / n}<\infty$. Thus by Lemma 2.1 we have that

$$
\sup _{k}\left\|\mu_{n} \lambda_{k}^{-1} A_{n k}\right\|^{1 / k}=L(n)<\infty
$$

for each $n \geq 1$. Now we denote

$$
f_{n}(\bar{x})=\left\|\sum_{k=1}^{\infty} \mu_{n} A_{n k} x_{k}\right\|^{1 / n},
$$

and we see that $\left(f_{n}(\bar{x})\right)$ is pointwise bounded. Further if $P_{\lambda}(\bar{x})=\sup _{k}\left\|\lambda_{k} x_{k}\right\|^{1 / k}<\eta$ then $f_{n}$ is continuous functional on $\Gamma(X, \lambda)$, for each $n \geq 1$. Moreover $\Gamma(X, \lambda)$ is complete linear metric space therefore by uniform boundedness principle (Osgood theorem) we can find $L>0$ and a closed sphere $S[\bar{\theta}, \delta]$ in $\Gamma(X, \lambda)$ such that

$$
\left|f_{n}(\bar{x})\right| \leq L
$$

for all $n \geq 1$ and for all $\bar{x} \in S[\bar{\theta}, \delta]$, or

$$
\left\|\sum_{k=1}^{\infty} \mu_{n} A_{n k} x_{k}\right\| \leq L^{n}
$$

for all $n \geq 1$ and for all $\bar{x} \in S[\bar{\theta}, \delta]$. Now take $z \in S$ arbitrary and consider the sequence

$$
\bar{x}=\left(\theta, \theta, \ldots, \theta, \delta^{k} \lambda_{k}^{-1} z, \theta, \theta, \ldots\right)
$$

where $\delta^{k} \lambda_{k}^{-1} z$ has the entry at $k^{\text {th }}$ place. Thus $\bar{x} \in S[\bar{\theta}, \delta]$ and so from (2.3) if follows that

$$
\left\|\mu_{n} \delta^{k} \lambda_{k}^{-1} A_{n k} z\right\| \leq L^{n}
$$

for all $n \geq 1, k \geq 1$ and $z \in S$, which implies that

$$
\left\|\mu_{n} \lambda_{k}^{-1} A_{n k}\right\| \leq \frac{L^{n}}{\delta^{k}}=M^{n+k}
$$

for all $n \geq 1$ and $k \geq 1$, where $M=\max \left(L, \delta^{-1}\right)$. This completes the proof.

\section{Characterization of $\left(l_{1}(X, \lambda), \Gamma(Y, \mu)\right)$}

In this section we determine the necessary and sufficient conditions for a matrix to map $l_{1}(X, \lambda)$ into $\Gamma(Y, \mu)$.

Definition 3.1. If $\gamma$ and $\rho$ are any two classes of scalar sequences then we define

$$
\begin{aligned}
& \gamma(X)=\left\{\bar{x}=\left(x_{k}\right): x_{k} \in X, \quad \text { such that }\left(\left\|x_{k}\right\|\right) \in \gamma\right\}, \\
& \gamma(X, \lambda)=\left\{\bar{x}=\left(x_{k}\right): x_{k} \in X, \quad \text { such that }\left(\lambda_{k} x_{k}\right) \in \gamma(X)\right\} .
\end{aligned}
$$


Similarly $\rho(X)$ and $\rho(X, \lambda)$ are defined.

Lemma 3.2. $A=\left(A_{n k}\right) \in(\gamma(X), \rho(Y))$ iff $B=\left(\mu_{n}^{-1} \lambda_{k} A_{n k}\right) \in(\gamma(X, \lambda), \rho(Y, \mu))$.

Proof. Let $\left(A_{n k}\right) \in(\gamma(X), \rho(Y))$ then for every $\bar{x}=\left(x_{k}\right) \in \gamma(X), y_{n}=\sum_{k=1}^{\infty} A_{n k} x_{k}$ exists for each $n$ and $\bar{y}=\left(y_{n}\right) \in \rho(Y)$. Now consider $\bar{u}=\left(u_{n}\right) \in \gamma(X, \lambda)$ and its transform $\bar{v}=B \bar{u}, v_{n}=\sum_{k=1}^{\infty} \mu_{n}^{-1} \lambda_{k} A_{n k} u_{k}$. Thus $\left(\lambda_{k} u_{k}\right) \in \gamma(X)$ and so $\left(y_{n}\right)=$ $\left(\sum_{k=1}^{\infty} A_{n k} \lambda_{k} u_{k}\right)_{n=1}^{\infty} \in \rho(Y)$ which implies that $\left(\mu_{n}^{-1} y_{n}\right) \in \rho(Y, \mu)$ and hence $\bar{v}=\left(v_{n}\right) \in$ $\rho(Y, \mu)$. Similarly the converse may be proved. The proof is now complete.

Applying Lemma 3.2 to Theorem 4.9 of $[7$, p.53] we easily get

Lemma 3.3. Let $1 \leq p<\infty$. Then $A=\left(A_{n k}\right) \in\left(l_{1}(X, \lambda), l_{p}(Y, \mu)\right)$ if and only if

$$
\sup \sum_{n=1}^{\infty}\left\|\mu_{n} \lambda_{k}^{-1} A_{n k} z\right\|^{p}<\infty,
$$

where the supremum is taken over all $z \in S$ and all $k \geq 1$.

Following Lemma can easily be obtained by taking $p_{k}=1$ in Theorem 3.2 of [9] and considering all $A_{k} \in B(X, Y)$ (cf. [7, p.26]).

Lemma 3.4. Let $A_{k} \in B(X, Y)$ for all $k \geq 1$. Then $\left(A_{k}\right) \in l_{1}^{\beta}(X, \lambda)$ if and only if $\sup _{k}\left\|\lambda_{k}^{-1} A_{k}\right\|<\infty$.

Theorem 3.5. $A=\left(A_{n k}\right) \in\left(l_{1}(X, \lambda), \Gamma(Y, \mu)\right)$ if and only if

(i) $\sup _{k}\left\|\lambda_{k}^{-1} A_{n k}\right\|<\infty$, for each $n \geq 1$, and

(ii) $\left\|\mu_{n} \lambda_{k}^{-1} A_{n k}\right\|^{1 / n} \rightarrow 0$ as $n \rightarrow \infty$ uniformly in $k$.

Proof. Sufficient part of the theorem can be proved easily with the help of Lemma 3.4 .

Let $A=\left(A_{n k}\right) \in\left(l_{1}(X, \lambda), \Gamma(Y, \mu)\right)$. The necessity of (i) follows easily by applying Lemma 3.4 to the fact that $y_{n}=\sum_{k=1}^{\infty} A_{n k} x_{k}$ exists for each $\bar{x}=\left(x_{k}\right) \in l_{1}(X, \lambda)$. For the necessity of (ii) suppose $A=\left(A_{n k}\right) \in\left(l_{1}(X, \lambda), \Gamma(Y, \mu)\right)$ but $\left\|\mu_{n} \lambda_{k}^{-1} A_{n k}\right\|^{1 / n} \nrightarrow 0$ as $n \rightarrow \infty$, uniformly in $k$. Thus for an $\varepsilon>0$ and a given $N$ there exist an $n>N$ and a $k \geq 1$ such that

$$
\left\|\mu_{n} \lambda_{k}^{-1} A_{n k}\right\|^{1 / n} \geq \varepsilon
$$

Further since $\Gamma(Y, \mu) \subset l_{1}(Y, \mu)$ therefore $\left(A_{n k}\right) \in\left(l_{1}(X, \lambda), l_{1}(Y, \mu)\right)$ and so by Lemma 3.3 , taking $p=1$ therein, we get

$$
\sup \sum_{k=1}^{\infty}\left\|\mu_{n} \lambda_{k}^{-1} A_{n k} z\right\|<\infty,
$$

where the supremum is taken over all $z \in S$ and all $k \geq 1$. Thus there exists $L>0$ such that

$$
\left\|\mu_{n} \lambda_{k}^{-1} A_{n k} z\right\| \leq \frac{L}{2}
$$


for all $n \geq 1, k \geq 1$ and $z \in S$ and so for each $n \geq 1$

$$
V_{n}=\sup _{k}\left\|\mu_{n} \lambda_{k}^{-1} A_{n k}\right\| \leq \frac{L}{2}
$$

Now by considering the sequences $\bar{x}=(\theta, \theta, \theta, \ldots \theta, z, \theta, \theta, \ldots)$ in $l_{1}(X, \lambda)$ where $z \in X$ is at the $k^{\text {th }}$ place, $k \geq 1$, we get that

$$
\left\|\mu_{n} \lambda_{k}^{-1} A_{n k} z\right\|^{1 / n} \rightarrow 0 \text { as } n \rightarrow \infty, \text { for each } k \text { and for each } z \text {. }
$$

Thus in view of (3.1) we may choose $n(1)$ and $k(1)$ such that

$$
\left\|\mu_{n(1)} \lambda_{k(1)}^{-1} A_{n(1) k(1)}\right\|^{1 / n(1)} \geq \varepsilon
$$

and so there exists $z_{1} \in S$ such that

$$
\left\|\mu_{n(1)} \lambda_{k(1)}^{-1} A_{n(1) k(1)} z_{1}\right\| \geq \frac{1}{2^{n(1)}}\left\|\mu_{n(1)} \lambda_{k(1)}^{-1} A_{n(1) k(1)}\right\| .
$$

Next in view of $(3.1)$ and $(3.3)$ we choose $n(2)>n(1)$ sufficiently large and $k(2)>k(1)$ such that

$$
\begin{aligned}
& \frac{L}{2^{n(2)}}<\left[\frac{\varepsilon}{8}\right]^{n(1)} \\
& \mu_{n(2)} \lambda_{k(2)}^{-1} A_{n(2) k(2)} \|^{1 / n(2)} \geq \varepsilon, \quad \text { and } \\
& \left\|\mu_{n(2)} \lambda_{k(1)}^{-1} A_{n(2) k(1)}\right\|^{1 / n(2)}<\frac{\varepsilon}{16} .
\end{aligned}
$$

Now we select $z_{2} \in S$ such that

$$
\left\|\mu_{n(2)} \lambda_{k(2)} A_{n(2) k(2)} z_{2}\right\| \geq \frac{1}{2^{n(2)}}\left\|\mu_{n(2)} \lambda_{k(2)}^{-1} A_{n(2) k(2)}\right\| .
$$

Further in view of (3.1) and (3.3) we choose $n(3)>n(2)$ sufficiently large and $k(3)>$ $k(2)$ such that

$$
\begin{aligned}
& \frac{L}{2^{n(3)}}<\left[\frac{\varepsilon}{16}\right]^{n(2)} \\
& \left\|\mu_{n(3)} \lambda_{k(3)}^{-1} A_{n(3) k(3)}\right\|^{1 / n(3)} \geq \varepsilon,
\end{aligned}
$$

and

$$
\left\{\begin{array}{l}
\left\|\mu_{n(3)} \lambda_{k(2)}^{-1} A_{n(3) k(2)} z_{2}\right\|^{1 / n(3)}<\frac{\varepsilon}{24} \\
\left\|\mu_{n(3)} \lambda_{k(1)}^{-1} A_{n(3) k(1)} z_{1}\right\|^{1 / n(3)}<\frac{\varepsilon}{24}
\end{array}\right.
$$

Now we can select $z_{3} \in S$ such that

$$
\left\|\mu_{n(3)} \lambda_{k(3)}^{-1} A_{n(3) k(3)} z_{3}\right\| \geq \frac{1}{2^{n(3)}}\left\|\mu_{n(3)} \lambda_{k(3)}^{-1} A_{n(3) k(3)}\right\| .
$$


Again by (3.1) and (3.3) we can choose $n(4)>n(3)$ sufficiently large and $k(4)>k(3)$, such that

$$
\begin{aligned}
& \frac{L}{2^{n(4)}}<\left[\frac{\varepsilon}{24}\right]^{n(3)} \\
& \left\|\mu_{n(4)} \lambda_{k(4)}^{-1} A_{n(4) k(4)}\right\|^{1 / n(4)} \geq \varepsilon, \quad \text { and } \\
& \left\{\begin{array}{l}
\left\|\mu_{n(4)} \lambda_{k(3)}^{-1} A_{n(4) k(3)} z_{3}\right\|^{1 / n(4)}<\frac{\varepsilon}{32} \\
\left\|\mu_{n(4)} \lambda_{k(2)}^{-1} A_{n(4) k(2)} z_{2}\right\|^{1 / n(4)}<\frac{\varepsilon}{32} \\
\left\|\mu_{n(4)} \lambda_{k(1)}^{-1} A_{n(4) k(1)} z_{1}\right\|^{1 / n(4)}<\frac{\varepsilon}{32}
\end{array}\right.
\end{aligned}
$$

We take $z_{4} \in S$ such that

$$
\left\|\mu_{n(4)} \lambda_{k(4)}^{-1} A_{n(4) k(4)} z_{4}\right\| \geq \frac{1}{2^{n(4)}}\left\|\mu_{n(4)} \lambda_{k(4)}^{-1} A_{n(4) k(4)}\right\|,
$$

and so on.

We define

$$
\begin{aligned}
x_{k} & =\frac{1}{2^{n(j)}} z_{j}, \quad k=k(j), j=1,2,3 \ldots, \text { and } \\
& =\theta, \quad \text { otherwise. }
\end{aligned}
$$

Now taking into consideration (3.2), (3.4), (3.5) and (3.6) we get that

$$
\begin{aligned}
\left\|\mu_{n(1)} y_{n(1)}\right\|^{1 / n(1)} & \geq\left\|\mu_{n(1)} \lambda_{k(1)}^{-1} A_{n(1) k(1)} \frac{1}{2^{n(1)}} z_{1}\right\|^{1 / n(1)}-\left\|\sum_{j=2}^{\infty} \mu_{n(1)} \lambda_{k(j)}^{-1} A_{n(1) k(j)} x_{k(j)}\right\|^{1 / n(1)} \\
& >\frac{1}{2} \cdot \frac{1}{2} \cdot \varepsilon-\frac{\varepsilon}{8}=\frac{\varepsilon}{8}
\end{aligned}
$$

because by (3.4) and (3.5) we have

$$
\begin{aligned}
\left\|\mu_{n(1)} \lambda_{k(1)}^{-1} A_{n(1) k(1)} \frac{1}{2^{n(1)}} z_{1}\right\|^{1 / n(1)} & \geq \frac{1}{2} \cdot \frac{1}{2}\left\|\mu_{n(1)} \lambda_{k(1)}^{-1} A_{n(1) k(1)}\right\|^{1 / n(1)} \\
& \geq \frac{1}{2} \cdot \frac{1}{2} \varepsilon
\end{aligned}
$$

and by (3.2) and (3.6) we have

$$
\begin{aligned}
\left\|\sum_{j=2}^{\infty} \mu_{n(1)} \lambda_{k(j)}^{-1} A_{n(1) k(j)} x_{k(j)}\right\| & \leq \sum_{j=2}^{\infty}\left\|\mu_{n(1)} \lambda_{k(j)}^{-1} A_{n(1) k(j)} \frac{1}{2^{n(j)}} z_{j}\right\| \\
& \leq V_{n(1)} \sum_{j=2}^{\infty} \frac{1}{2^{n(j)}} \leq \frac{L}{2^{n(2)}}<\left[\frac{\varepsilon}{8}\right]^{n(1)}
\end{aligned}
$$

Further by (3.4), (3.9), (3.10) and (3.12) we get $\left\|\mu_{n(2)} Y_{n(2)}\right\|^{1 / n(2)} \geq\left\|\mu_{n(2)} \lambda_{k(2)}^{-1} A_{n(2) k(2)} \frac{1}{2^{n(2)}} z_{2}\right\|^{1 / n(2)}-\left\|\mu_{n(2)} \lambda_{k(1)}^{-1} A_{n(2) k(1)} x_{k(1)}\right\|^{1 / n(2)}$ 


$$
\begin{aligned}
& -\left\|\sum_{j=3}^{\infty} \mu_{n(2)} \lambda_{k(j)}^{-1} A_{n(2) k(j)} x_{k(j)}\right\|^{1 / n(2)} \\
> & \frac{1}{2} \cdot \frac{1}{2} \cdot \varepsilon-\frac{\varepsilon}{16}-\frac{\varepsilon}{16}=\frac{\varepsilon}{8}
\end{aligned}
$$

since (3.7) and (3.9) yield

$$
\begin{aligned}
\left\|\mu_{n(2)} \lambda_{k(2)}^{-1} A_{n(2) k(2)} \frac{1}{2^{n(2)}} z_{2}\right\|^{1 / n(2)} & \geq \frac{1}{2} \cdot \frac{1}{2}\left\|\mu_{n(2)} \lambda_{k(2)}^{-1} A_{n(2) k(2)}\right\|^{1 / n(2)} \\
& \geq \frac{1}{2} \cdot \frac{1}{2} \varepsilon
\end{aligned}
$$

(3.8) yields

$$
\begin{aligned}
\left\|\mu_{n(2)} \lambda_{k(1)}^{-1} A_{n(2) k(1)} x_{k(1)}\right\| & =\left\|\mu_{n(2)} \lambda_{k(1)}^{-1} A_{n(2) k(1)} \frac{1}{2^{n(1)}} z_{1}\right\| \\
& <\frac{L}{2^{n(1)}}\left[\frac{\varepsilon}{16}\right]^{n(2)}<\left[\frac{\varepsilon}{16}\right]^{n(2)}
\end{aligned}
$$

and (3.2) and (3.10) yield,

$$
\begin{aligned}
\left\|\sum_{j=3}^{\infty} \mu_{n(2)} \lambda_{k(j)}^{-1} A_{n(2) k(j)} x_{k(j)}\right\| & \leq \sum_{j=3}^{\infty}\left\|\mu_{n(2)} \lambda_{k(j)}^{-1} A_{n(2) k(j)}\right\| \frac{1}{2^{n(j)}} \\
& \leq V_{n(2)} \sum_{j=3}^{\infty} \frac{1}{2^{n(j)}} \leq \frac{L}{2^{n(3)}}<\left[\frac{\varepsilon}{16}\right]^{n(2)} .
\end{aligned}
$$

Similarly by (3.2), (3.11), (3.12), (3.13) and (3.14) we get

$$
\begin{aligned}
\left\|\mu_{n(3)} y_{n(3)}\right\|^{1 / n(3)} \geq & \left\|\mu_{n(3)} \lambda_{k(3)}^{-1} A_{n(3) k(3)} \frac{1}{2^{n(3)}} z_{3}\right\|^{1 / n(3)}-\left\|\mu_{n(3)} \lambda_{k(2)}^{-1} A_{n(3) k(2)} x_{k(2)}\right\|^{1 / n(3)} \\
& -\left\|\mu_{n(3)} \lambda_{k(1)}^{-1} A_{n(3) k(1)} x_{k(1)}\right\|^{1 / n(3)}-\left\|\sum_{j=4}^{\infty} \mu_{n(3)} \lambda_{k(j)}^{-1} A_{n(3) k(j)} x_{k(j)}\right\|^{1 / n(3)} \\
> & \frac{1}{2} \cdot \frac{1}{2} \cdot \varepsilon-\frac{\varepsilon}{24}-\frac{\varepsilon}{24}-\frac{\varepsilon}{24}=\frac{\varepsilon}{8},
\end{aligned}
$$

since by (3.11) and (3.13)

$$
\left\|\mu_{n(3)} \lambda_{k(3)}^{-1} A_{n(3) k(3)} \frac{1}{2^{n(3)}} z_{3}\right\|^{1 / n(3)} \geq \frac{1}{2} \cdot \frac{1}{2}\left\|\mu_{n(3)} \lambda_{k(3)}^{-1} A_{n(3) k(3)}\right\|^{1 / n(3)} \geq \frac{1}{2} \cdot \frac{1}{2} \varepsilon,
$$

by (3.12)

$$
\begin{aligned}
\left\|\mu_{n(3)} \lambda_{k(2)}^{-1} A_{n(3) k(2)} x_{k(2)}\right\| & =\frac{1}{2^{n(2)}}\left\|\mu_{n(3)} \lambda_{k(2)}^{-1} A_{n(3) k(2)} z_{2}\right\|^{1 / n(3)} \\
& \leq \frac{1}{2^{n(2)}}\left[\frac{\varepsilon}{24}\right]^{n(3)}<\left[\frac{\varepsilon}{24}\right]^{n(3)}
\end{aligned}
$$


and similarly

$$
\left\|\mu_{n(3)} \lambda_{k(1)}^{-1} A_{n(3) k(1)} x_{k(1)}\right\|<\left[\frac{\varepsilon}{24}\right]^{n(3)}
$$

and by (3.2) and (3.14)

$$
\begin{aligned}
\left\|\sum_{j=4}^{\infty} \mu_{n(3)} \lambda_{k(j)}^{-1} A_{n(3) k(j)} x_{k(j)}\right\| & \leq \sum_{j=4}^{\infty}\left\|\mu_{n(3)} \lambda_{k(j)}^{-1} A_{n(3) k(j)}\right\| \frac{1}{2^{n(j)}} \\
& \leq V_{n(3)} \sum_{j=4}^{\infty} \frac{1}{2^{n(j)}} \leq \frac{L}{2^{n(4)}}<\left[\frac{\varepsilon}{24}\right]^{n(3)}
\end{aligned}
$$

Thus proceeding along the above lines we see that there exist sequences $(n(j)),(k(j))$ of integers and $\bar{x}=\left(x_{k}\right) \in l_{1}(X, \lambda)$ defined as above such that

$$
\left\|\mu_{n(j)} y_{n(j)}\right\|^{1 / n(j)}>\frac{\varepsilon}{8} ; \quad j=1,2,3 \ldots
$$

that is $\bar{y}=\left(y_{n}\right) \notin \Gamma(Y, \mu)$, which is a contradiction. This completes the proof.

\section{Characterization of $B\left(c_{0}(X, \lambda), c_{0}(Y, \mu)\right)$}

In this section we obtain necessary and sufficient conditions for a matrix of linear operators to map $c_{0}(X, \lambda)$ into $c_{0}(Y, \mu)$ and as an application of this result we characterize $B\left(c_{0}(X, \lambda), c_{0}(Y, \mu)\right)$, the Banach space of all bounded linear operators from the Banach space $c_{0}(X, \lambda)$ into the Banach space $c_{0}(Y, \mu)$ by obtaining matrix representation $\left(A_{n k}\right)$ of each $A \in B\left(c_{0}(X, \lambda), c_{0}(Y, \mu)\right)$.

From Theorem 3.3 [8] by taking $p_{k}=1$ and $A_{k} \in B(X, Y)$ for all $k$, we easily get:

Lemma 4.1. $\left(A_{k}\right) \in c_{0}^{\beta}(X, \lambda)$ if and only if $\left\|\left(\lambda_{k}^{-1} A_{k}\right)\right\|<\infty$.

Definition 4.2. A normed space $X$-valued sequence space $(E(X), \mathcal{J})$ equipped with the linear topology $\mathcal{J}$ is said to be a $G K$-space if map $P_{n}: E(X) \rightarrow X, P_{n}(\bar{x})=x_{n}$, is continuous for each $n$. A $G K$-space is said to be a $G A K$-space if for each $\bar{x}=\left(x_{k}\right)$ in $E(X), s_{n}(\bar{x}) \rightarrow \bar{x}$ as $n \rightarrow \infty$ with respect to $\mathcal{J}$, where $s_{n}(\bar{x})=\left(x_{1}, x_{2}, x, \ldots x_{n}, \theta, \theta, \ldots\right)$. Further $(E(X), \mathcal{J})$ is said to be a $G C$-space if $R_{n}: X \rightarrow E(X), R_{n}(x)=\delta_{n}(x)$, is continuous for each $n$, where $\delta_{n}(x)=(\theta, \theta, \ldots, \theta, x, \theta \ldots), x$ at $n^{\text {th }}$ place.

Above definitions are the generalizations of $K-, A K$ - and $C$-spaces of scalar sequences (see [4]).

Following lemma can easily be proved:

Lemma 4.3. The Banach space $\left(c_{0}(X, \lambda),\|\cdot\|_{\lambda}\right)$ is a $G K-, G A K$-and $G C$-space.

Theorem 4.4. (a) $\left(A_{n k}\right) \in\left(c_{0}(X, \lambda), c_{0}(Y, \mu)\right)$ if and only if (i) $\sup _{n}\left\|\left(\mu_{n} \lambda_{k}^{-1} A_{n k}\right)\right\|=H<\infty$; and 
(ii) $\lim _{n} \mu_{n} A_{n k}=\theta$, for each $k \geq 1$.

(b) $A \in B\left(c_{0}(X, \lambda), c_{0}(Y, \mu)\right)$ if and only if $A$ is a matrix transformation $\left(A_{n k}\right)$ satisfying

(i) and $($ ii $)$. Moreover

$$
\|A\|=\sup _{n}\left\|\left(\mu_{n} \lambda_{k}^{-1} A_{n k}\right)\right\|
$$

Proof. (a) Let $\left(A_{n k}\right) \in\left(c_{0}(X, \lambda), c_{0}(Y, \mu)\right)$. Necessity of (ii) follows easily by considering the sequences $(\theta, \theta, \theta, \ldots \theta, x, \theta, \ldots), x \in X$ at $k^{t h}$ place, $k \geq 1$. We note that $c_{0}(X, \lambda)$ is a Banach space with $\|\bar{x}\|_{\lambda}=\sup _{k}\left\|\lambda_{k} x_{k}\right\|, \bar{x}=\left(x_{k}\right) \in c_{0}(X, \lambda)$. Thus for $\bar{x} \in c_{0}(X, \lambda)$ and each $n \geq 1$ if we define

$$
T_{n}(\bar{x})=\sum_{k=1}^{\infty} \mu_{n} A_{n k} x_{k}
$$

and

$$
T_{n, p}(\bar{x})=\sum_{k=1}^{p} \mu_{n} A_{n k} x_{k}, \quad p \geq 1,
$$

then for each $n, T_{n, p}(\bar{x}) \rightarrow T_{n}(\bar{x})$ in $Y$ as $p \rightarrow \infty$ for every $\bar{x} \in c_{0}(X, \lambda)$. Moreover for each $n$ and $p$

$$
\left\|T_{n, p}(\bar{x})\right\| \leq\left(\sum_{k=1}^{p}\left\|\mu_{n} \lambda_{k}^{-1} A_{n k}\right\|\right)\|\bar{x}\|_{\lambda}
$$

which shows that $T_{n, p}: c_{0}(X, \lambda) \rightarrow Y$ is a bounded linear operator. Hence by BanachSteinhaus theorem, $T_{n}: c_{0}(X, \lambda) \rightarrow Y$ is also a bounded linear operator for each $n \geq 1$.

Further the transform $\bar{y}=\left(y_{n}\right)$, where $y_{n}=\sum_{k=1}^{\infty} A_{n k} x_{k}$, of each $\bar{x}=\left(x_{k}\right) \in c_{0}(\bar{X}, \lambda)$ is in $c_{0}(Y, \mu)$ therefore $\left(T_{n}(\bar{x})\right)_{n=1}^{\infty}$ is bounded for each $\bar{x} \in c_{0}(X, \lambda)$. Again on application of Banach-Steinhaus theorem we get $L>0$ such that $\left\|T_{n}(\bar{x})\right\| \leq L\|\bar{x}\|_{\lambda}$, for all $n \geq 1$ and $\bar{x} \in c_{0}(X, \lambda)$. Now for any $n \geq 1$ if we consider $x_{1}, x_{2}, x_{3} \ldots x_{p} \in S, p \geq 1$, arbitrary then $\bar{x}=\left(\lambda_{1}^{-1} x_{1}, \lambda_{2}^{-1} x_{2}, \lambda_{3}^{-1} x_{3} \ldots \lambda_{p}^{-1} x_{p}, \theta, \theta \ldots\right) \in c_{0}(X, \lambda)$ with $\|\bar{x}\|_{\lambda} \leq 1$, and so we have

$$
\left\|\sum_{k=1}^{p} \mu_{n} \lambda_{k}^{-1} A_{n k} x_{k}\right\|=\left\|T_{n}(\bar{x})\right\| \leq L\|\bar{x}\|_{\lambda} \leq L,
$$

for all $x_{1}, x_{2}, \ldots x_{p} \in S, p \geq 1$. Thus taking supremum over these quantities we easily get

$$
\left\|\left(\mu_{n} \lambda_{k}^{-1} A_{n k}\right)\right\| \leq L, \quad \text { for every } n \geq 1,
$$

and hence

$$
\sup _{n}\left\|\left(\mu_{n} \lambda_{k}^{-1} A_{n k}\right)\right\| \leq L
$$

This proves the necessity of (i).

Let (i) and (ii) hold. Then by Lemma 4.1 it follows that for every $\bar{x} \in c_{0}(X, \lambda)$ and for each $n \geq 1, \sum_{k=1}^{\infty} A_{n k} x_{k}$ converges in $Y$. Now for $\bar{x} \in c_{0}(X, \lambda)$ and $\varepsilon>0$ there exists $K$ such that $\left\|\lambda_{k} x_{k}\right\|<\varepsilon$, for every $k \geq K$ and in view of (ii) there exists $N$ such that

$$
\sum_{k=1}^{K-1}\left\|\mu_{n} A_{n k} x_{k}\right\|<\varepsilon, \quad \text { for all } n \geq N,
$$


hence

$$
\begin{aligned}
\left\|\mu_{n} y_{n}\right\| & \leq \sum_{k=1}^{K-1}\left\|\mu_{n} A_{n k} x_{k}\right\|+\left\|\sum_{k=K}^{\infty} \mu_{n} \lambda_{k}^{-1} A_{n k}\left(\lambda_{k} x_{k}\right)\right\| \\
& \leq \sum_{k=1}^{K-1}\left\|\mu_{n} A_{n k} x_{k}\right\|+\| R_{K}\left(\mu_{n} \lambda_{k}^{-1} A_{n k}\left\|\sup _{k \geq K}\right\| \lambda_{k} x_{k} \|\right. \\
& <(1+H) \varepsilon,
\end{aligned}
$$

for all $n \geq N$, as $\left\|R_{K}\left(\mu_{n} \lambda_{k}^{-1} A_{n k}\right)\right\| \leq\left\|\left(\mu_{n} \lambda_{k}^{-1} A_{n k}\right)\right\| \leq H$, i.e., $\left(y_{n}\right) \in c_{0}(Y, \mu)$, which proves the sufficiency of (i) and (ii).

(b) Let $A: c_{0}(X, \lambda) \rightarrow c_{0}(Y, \mu)$ be a bounded linear operator, and consider $A_{n k} x=$ $P_{n}^{Y} o A o R_{k}^{X}(x)$. Clearly $P_{n}^{Y}: c_{0}(Y, \mu) \rightarrow Y$, defined by $P_{n}^{Y}(\bar{y})=y_{n}$ is linear and bounded as $c_{0}(Y, \mu)$ is a $G K$-space. Again $R_{k}^{X}: X \rightarrow c_{0}(X, \lambda)$ defined by $R_{k}^{X}(x)=$ $(\theta, \theta, \ldots, \theta, x, \theta, \ldots), x$ at $k^{\text {th }}$ place. is linear and bounded since $c_{0}(X, \lambda)$ is a $G C$-space. Thus $A_{n k}: X \rightarrow Y$ is linear and bounded for each $n \geq 1$ and $k \geq 1$. Further we note that $\left(c_{0}(X, \lambda),\|\cdot\|_{\lambda}\right)$ is a $G A K$-space, so for each $\bar{x} \in c_{0}(X, \lambda), s_{p}(\bar{x}) \rightarrow \bar{x}$ in $c_{0}(X, \lambda)$. Thus if $A(\bar{x})=\left(y_{n}\right)$ then we have

$$
\begin{aligned}
y_{n} & =P_{n}^{Y}(A(\bar{x}))=\left(P_{n}^{Y} o A\right)\left(\lim _{p}\left(s_{p}(\bar{x})\right)\right. \\
& =P_{n}^{Y} o\left(\lim _{p} A\left(\sum_{k=1}^{p} R_{k}^{X}\left(x_{k}\right)\right)\right) \\
& =\lim _{p} \sum_{k=1}^{p} P_{n}^{Y} o A o R_{k}^{X}\left(x_{k}\right)=\sum_{k=1}^{\infty} A_{n k} x_{k}
\end{aligned}
$$

which shows that $A$ determines the matrix $\left(A_{n k}\right)$, where $A_{n k} \in B(X, Y)$, mapping $c_{0}(X, \lambda)$ into $c_{0}(Y, \mu)$ hence it will satisfy (i) and (ii).

Conversely $\left(A_{n k}\right) \in\left(c_{0}(X, \lambda), c_{0}(Y, \mu)\right)$ satisfies (i) and (ii) and so clearly it determines a linear operator $A: c_{0}(X, \lambda) \rightarrow c_{0}(Y, \mu)$ such that

$$
A(\bar{x})=\left(\sum_{k=1}^{\infty} A_{n k} x_{k}\right)_{n=1}^{\infty}
$$

Further

$$
\begin{aligned}
\|A(\bar{x})\|_{\mu} & =\sup _{n}\left\|\mu_{n} \sum_{k=1}^{\infty} A_{n k} x_{k}\right\| \\
& \leq \sup _{n}\left\|\left(\mu_{n} \lambda_{k}^{-1} A_{n k}\right)\right\| \sup _{k}\left\|\lambda_{k} x_{k}\right\| \leq H\|\bar{x}\|_{\lambda}
\end{aligned}
$$

shows that $A$ is bounded. 
Now whether the bounded linear operator $A: c_{0}(X, \lambda) \rightarrow c_{0}(Y, \mu)$ determines a matrix $A=\left(A_{n k}\right) \in\left(c_{0}(X, \lambda), c_{0}(Y, \mu)\right)$ or vice-verse, we have from what has been discussed above that

$$
\|A(\bar{x})\|_{\mu} \leq H\|x\|_{\lambda}
$$

and so

$$
\|A\| \leq H
$$

On the other hand in view of (i) for $\varepsilon>0$ there exists $m$ such that

$$
\left\|\left(\mu_{m} \lambda_{k}^{-1} A_{m k}\right)\right\|>H-\frac{\varepsilon}{2}
$$

and further there exist $p \geq 1$ and $x_{1}, x_{2}, \ldots x_{p} \in S$ such that

$$
\left\|\sum_{k=1}^{p} \mu_{m} \lambda_{k}^{-1} A_{m k} x_{k}\right\|>H-\frac{\varepsilon}{2}-\frac{\varepsilon}{2}=H-\varepsilon
$$

Now if we consider $\bar{x}=\left(\lambda_{1}^{-1} x_{1}, \lambda_{2}^{-1} x_{2}, \ldots \lambda_{p}^{-1} x_{p}, \theta, \theta, \ldots\right)$ in $c_{0}(X, \lambda)$ then $\|\bar{x}\|_{\lambda} \leq 1$ and

$$
\begin{aligned}
\|A(\bar{x})\|_{\mu} & =\sup _{n}\left\|\mu_{n} \sum_{k=1}^{p} A_{n k} \lambda_{k}^{-1} x_{k}\right\| \\
& \geq\left\|\mu_{m} \sum_{k=1}^{p} A_{m k} \lambda_{k}^{-1} x_{k}\right\|>H-\varepsilon
\end{aligned}
$$

i.e. $\|A\|>H-\varepsilon$, for $\varepsilon>0$ arbitrary, and so $\|A\| \geq H$. This together with (4.1) leads to

$$
\|A\|=H=\sup _{n}\left\|\left(\mu_{n} \lambda_{k}^{-1} A_{n k}\right)\right\| .
$$

This completes the proof.

Finally we note that the Theorems 3.1, 3.2 and 3.3 of Das and Chaudhary [1] can easily be deduced from our Theorem 4.2 .

\section{Acknowledgement}

This paper was written while J. K. Srivastava held a project (No F. 8-5/90 RBB-II, dated July 3, 1991) of the University Grants Commission and B. K. Srivastava worked in it as project assistant. The support by commission is very gratefully acknowledged.

We are also thankful to the referee for his suggestions to improve the presentation of the paper. 


\section{References}

[1] N. R. Das and A. Chaudhary, Matrix transformations of vector valued sequence spaces, Bull. Cal. Math. Soc. 84(1992), 47-54.

[2] V. G. Iyer, On the space of integral functions - I, J. Indian Math. Soc. (N. S.) 12(1948), 13-20.

[3] V. G. Iyer, On the space of integral functions - II, Quart. J. Math. Oxford (2), 1(1950), 86-96.

[4] P. K. Kamthan and M. Gupta, Sequence Spaces and Series, Marcel Dekker Inc, 1981.

[5] K. Chandrasekhara Rao, Matrix transformations of some sequence spaces, Pacific J. Math. 31(1969), 171-174.

[6] K. Chandrasekhara Rao, Matirx transformations of some sequence spaces - II, Glasgow Math. J. 11(1970), 162-166.

[7] I. J. Maddox, Infinite matrices of Operators, Lecture Notes in Mathematics, 786, SpringerVerlag, 1980.

[8] J. K. Srivastava and B. K. Srivastava, Generalized sequence space $c_{0}(X, \lambda, p)$, Indian J. Pure Appl. Math. 27(1996), 73-84.

[9] J. K. Srivastava and B. K. Srivastava, Generalized sequence space $l(X, \lambda, p), l_{\infty}(X, \lambda, p)$ and $c(X, \lambda, p)$, (submitted for publication).

Department of Mathematics \& Statistics, D. D. U. Gorakhpur University, GORAKHPUR 273009, INDIA. 